\title{
Síndrome metabólico en diálisis peritoneal
}

\author{
Francisco Cirera Segura - Jesús Lucas Martín Espejo
}

\section{Enfermeros \\ Unidad de Gestión Clínica Uro-Nefrológica \\ H.H.U.U. Virgen del Rocío. Sevilla}

\section{Resumen}

Introducción: El síndrome metabólico es una agrupación de factores de riesgo cardiovascular de origen metabólico.

Nos planteamos:

- Determinar la prevalencia del síndrome metabólico en diálisis peritoneal y ver su evolución.

- Valorar su influencia sobre la mortalidad.

- Revisar las intervenciones de Enfermería destinadas a prevenirlo.

Material y métodos: Se realizó un estudio descriptivo retrospectivo de 5 años.

Se utilizó la clasificación de criterios diagnósticos del síndrome metabólico propuesta por NCEP ATP III, modificada por Kam-Tao Li.

El resto de las variables se recogieron de la historia clínica.

Resultados: La muestra la compusieron 53 pacientes con una edad media de 62,02 años. El tiempo medio en diálisis peritoneal fue de 28,02 meses.

Dirección para la correspondencia:

Francisco Cirera Segura.

C/Ángel Ripoll Pastor, $\mathrm{n}^{\circ} 4-6^{\circ} \mathrm{A}$

41006 Sevilla

e.mail:paco.cirera@gmail.com

Se estudiaron los factores que describen el síndrome metabólico en diálisis peritoneal. No difirieron salvo en la glucemia $(p=0,012)$.

Los pacientes diagnosticados de síndrome metabólico aumentaron, sin significación $(p=0,18)$.

La supervivencia de los pacientes con síndrome metabólico fue menor aunque sin diferencia significativa $(p=0,990)$.

Discusión: El papel de Enfermería en la prevención y tratamiento de síndrome metabólico es fundamental, ya que podemos actuar sobre todos los factores.

El "Déficit de Conocimientos" debe evaluarse de forma sistemática para reforzar las intervenciones. Comprobamos la importancia de establecer diagnósticos de Enfermería y reevaluar intervenciones diseñadas para obtener los resultados esperados.

No hemos conseguido mejorar el síndrome metabólico lo que nos obliga a plantearnos actuaciones más efectivas, ya que:

- Tenemos un elevado porcentaje de pacientes con síndrome metabólico que aumenta al año.

- Tuvieron menor supervivencia, aunque sin significación.

- Debemos corregir los diagnósticos e intervenciones de Enfermería. 


\section{PALABRAS CLAVE: \\ - DIÁLISIS PERITONEAL \\ - SÍNDROME METABÓLICO \\ - ENFERMERÍA \\ - RIESGO CARDIOVASCULAR}

\section{Metabolic syndrome in peritoneal dialysis}

\section{Abstract}

Introduction: Metabolic syndrome is a combination of cardiovascular risk factors of metabolic origin.

We set out to:

- Determine the prevalence of metabolic syndrome in peritoneal dialysis and see how it evolved.

- Evaluate its influence on mortality.

- Review the Nursing interventions aimed at preventing it.

Material and methods: A 5-year retrospective descriptive study was carried out.

The metabolic syndrome diagnostic criteria classification proposed by NCEP ATP III, modified by Kam-Tao Li, was used.

The other variables were obtained from the clinical history.

Results: The sample was made up of 53 patients with an average age of 62.02 years. The average time on dialysis was 28.02 months.

The factors that describe metabolic syndrome in peritoneal dialysis were studied. They did not differ, except for glucaemia $(p=0.012)$.

The patients diagnosed with metabolic syndrome increased, without significance $(p=0.18)$.

The survival of patients with metabolic syndrome was lower, although without a significant difference $(p=0.990)$.
Discussion: The role of Nursing in the prevention and treatment of metabolic syndrome is fundamentally, as we can act on all the factors.

The "Knowledge Deficit" should be assessed systematically in order to reinforce the intervention. We found the importance of establishing Nursing diagnoses and re-assessing interventions designed to obtain the expected results.

We have not managed to improve metabolic syndrome, which forces us to consider more effective actions, because

- We have a high percentage of patients with metabolic syndrome, which increases every year.

- They had lower survival, although the difference was not significant.

- We should correct Nursing diagnoses and interventions.

\section{KEY WORDS:}

- PERITONEAL DIALYSIS

- METABOLIC SYNDROME

- NURSING

- CARDIOVASCULAR RISK

\section{Introducción}

Se han dado múltiples definiciones y nombres al síndrome metabólico (SM) $)^{1,2}$, aunque en definitiva, es una agrupación de factores de riesgo cardiovascular (FRCV) de origen metabólico (elevación de la presión arterial, alteración del perfil lipídico a expensas de un incremento de triglicéridos y colesterol LDL y descenso del colesterol HDL, hiperglucemia, y obesidad), que sumados incrementan de forma exponencial el riesgo cardiovascular ${ }^{2}$ y pueden conducir al desarrollo de diabetes mellitus ${ }^{3}$.

Aunque el SM no es un indicador absoluto de riesgo cardiovascular, conlleva un incremento de la morbi- 
mortalidad de origen ateroesclerótico ${ }^{4}$. Esto se ha confirmado en una revisión sistemática con su correspondiente metaanálisis de 38 estudios longitudinales que incluyó 172.573 individuos, de manera que aquéllos con SM tenían 1,78 veces más riesgo de padecer algún evento cardiovascular y de muerte $\mathrm{e}^{5-6}$.

En diálisis peritoneal (DP) el estudio del SM tiene una especial relevancia debido a las alteraciones metabólicas producidas por la sobrecarga de glucosa que inducen los líquidos de diálisis. Esta situación ha provocado que la Enfermería de DP, empiece a tomar conciencia de la importancia que tiene en el control de los FRCV, ya que su detección y tratamiento precoz podrían disminuir la mortalidad cardiovascular asociada al $\mathrm{SM}^{3}$.

Los objetivos que nos hemos planteado para este trabajo son:

- Determinar la prevalencia del SM al inicio del programa de DP y ver su evolución al año.

- Valorar la influencia del SM sobre la mortalidad en nuestros pacientes.

- Revisar las intervenciones de Enfermería destinadas a prevenir la instauración del SM.

\section{Material y métodos}

Se realizó un estudio descriptivo retrospectivo de 5 años de evolución, cuya población fueron todos los pacientes incidentes en DP desde el 1 de enero de 2005 al 31 de diciembre de 2010 en nuestro centro $(n=119)$. Los criterios de exclusión fueron: pacientes menores de 18 años, menos de 1 año en el programa de DP $(n=61)$ y pacientes con enfermedades sistémicas graves (cirrosis, neoplasias, EPOC...) ( $n=4)$. Se retiró un paciente más por no tener datos analíticos en las fechas de corte del estudio. La muestra por lo tanto, estuvo compuesta por 53 pacientes; 28 hombres y 25 mujeres.

Habitualmente se han utilizado cinco tipos de clasificación de criterios diagnósticos para el SM, aunque la más aceptada es la que propuso la NCEP ATP III7, modificada posteriormente por Kam-Tao Li et al ${ }^{1}$ para adecuarla a pacientes en DP. Mediante esta clasificación, que es la utilizada en este estudio, se diagnostica el SM cuando se reúnen 3 o más de los siguientes criterios: Obesidad (I.M.C. $\left.>30 \mathrm{Kg} / \mathrm{m}^{2}\right)^{8}$, Triglicéridos elevados ( $\geq 150 \mathrm{mg} / \mathrm{dL}$ ), colesterol H.D.L. bajo ( $<40 \mathrm{mg} /$ $\mathrm{dL}$ en hombres, $\mathrm{y}<50 \mathrm{mg} / \mathrm{dL}$ en mujeres), aumento de la presión arterial $(\geq 130 \mathrm{~mm}$ de $\mathrm{Hg}$ sistólica $0 \geq 85$ $\mathrm{mm}$ de $\mathrm{Hg}$ diastólica) o hipertenso en tratamiento, alteración de la glucemia en ayunas ( $\geq 100 \mathrm{mg} / \mathrm{dL}$ ) 0 diabético en tratamiento.

Además se recogieron las siguientes variables de la historia clínica digital al inicio y al año de permanencia en el programa de DP:

- Demográficas: edad, sexo, patología de base ${ }^{9}$ peso.

- Datos analíticos: urea, creatinina, glucemia, fósforo, calcio, hemoglobina, parathormona (iPTH), proteína $C$ reactiva ( $P C R$ ), albúmina, colesterol total, colesterol LDL, proteínas totales y diuresis residual.

- Otras variables: tiempo en DP, modalidad de tratamiento, dosis de diálisis (Kt/V), tratamiento farmacológico con hipolipemiantes e hipotensores, exitus de etiología cardiovascular.

Para realizar la estadística descriptiva de las variables del estudio usamos frecuencias absolutas y relativas en el caso de las variables cualitativas. Las cuantitativas según si su distribución fuera normal o no, se resumieron mediante la media y la desviación estándar o la mediana y el rango intercuartílico respectivamente. Realizamos la comprobación de los grupos del estudio mediante el test de McNemar o el test de Wilcoxon, y para la comparación de variables cuantitativas la T de Student para datos apareados. El estudio de supervivencia se realizó mediante curvas de Kaplan-Meier y se compararon con el test de Log-Rank (Mantel-Cox).

El análisis se realizó con el paquete estadístico IBM SPSS Statistics 19.0, estableciendo el nivel de significación en $p<0,05$.

\section{Resultados}

La muestra la compusieron un total 53 pacientes, 28 hombres y 25 mujeres, con una edad media de 62,02 $+15,33$ años al iniciar DP. El $66 \%(n=35)$ estaban en diálisis peritoneal continua ambulatoria (DPCA) y $34 \%$ $(n=18)$ en diálisis peritoneal automática (DPA). El tiempo medio en DP fue de 28,02 $\pm 12,88$ (12-55) meses. La etiología de la ERC se muestra en la tabla 1. 


\begin{tabular}{|l|c|}
\hline & $\begin{array}{c}\text { Porcentaje válido } \\
\text { (Frecuencia) }\end{array}$ \\
\hline No Filiada & $22,6 \%(n=12)$ \\
\hline Enfermedades Vasculares & $18,9 \%(n=10)$ \\
\hline Nefropatía Diabética & $17,0 \%(n=9)$ \\
\hline Pielonefritis/Nefritil Intersticial & $15,1 \%(n=8)$ \\
\hline Glomerulonefritis & $11,3 \%(n=6)$ \\
\hline Enfermedades Sistémicas & $7,5 \%(n=4)$ \\
\hline Enfermedad Poliquística & $3,8 \%(n=2)$ \\
\hline Enfermedades Hereditarias/Congénitas & $1,9 \%(n=1)$ \\
\hline Otras & $1,9 \%(n=1)$ \\
\hline
\end{tabular}

*Códigos EDTA 1994-1995 y su agrupación, extraídos del Informe 2006. Módulo Básico. Subsistema de Insuficiencia Renal Crónica. Servicio Andaluz de Salud. Junta de Andalucía

Tabla 1: Etiología de la Enfermedad Renal Crónica*
El resto de las características de la muestra al inicio del estudio se presentan en la tabla 2.

Los factores que describen el SM se recogen en la tabla 3 que muestra de forma individual cada factor al inicio y al año en el programa en DP. La situación basal no difirió de la del primer año, salvo en la glucemia donde existieron diferencias estadísticamente significativas ( $p=0,012)$. De igual forma se estudiaron los pacientes con diagnóstico establecido de SM, que habían aumentado aunque no de forma significativa $(p=0,18)$.

El 28,3\% ( $n=15)$ de los pacientes era diabético antes de iniciar DP. El 66,7\% ( $n=10)$ eran varones y el $33,3 \%(n=5)$ mujeres. La etiología de la ERC fue una

\begin{tabular}{|c|c|c|}
\hline & Totales & $\mathbf{n}$ \\
\hline \multicolumn{3}{|l|}{ DATOS CLÍNICOS } \\
\hline Pacientes & $n=53$ & \multirow{4}{*}{53} \\
\hline Edad (años) & $62,02(15,33)$ & \\
\hline Tiempo en D.P. (meses) & $28,02(12,88)$ & \\
\hline D.P.C.A.:D.P.A. & $35: 18$ & \\
\hline $\mathrm{Kt} / \mathrm{V}$ Total & $2,56(0,73)$ & 52 \\
\hline Diuresis 24 horas ( $\mathrm{mL})$ & $1911,60(647,74)$ & \multirow{2}{*}{53} \\
\hline Hipertensión (Sí/No) & $39: 14$ & \\
\hline \multicolumn{3}{|c|}{ DATOS ANTROPOMÉTRICOS } \\
\hline Peso $(\mathrm{Kg})$ & $71,13(13,49)$ & \multirow{2}{*}{53} \\
\hline I.M.C. $\left(\mathrm{Kg} / \mathrm{m}^{2}\right)$ & $27,13(4,91)$ & \\
\hline \multicolumn{3}{|l|}{ DATOS ANALÍTICOS } \\
\hline Hemoglobina (mg/dL) & $112,57(17,76)$ & \multirow{4}{*}{53} \\
\hline Creatinina (mg/dL) & $7,8(1,8)$ & \\
\hline Urea (mg/dL) & $179,02(48,04)$ & \\
\hline Glucosa (mg/dL) & $91(21,30)$ & \\
\hline Calcio (mg/dL) & $9,06(09,96)$ & 52 \\
\hline Fósforo (mg/dL) & $5,68(1,34)$ & 52 \\
\hline iPTH $(p g / m L)$ & $270,84(222,27)$ & 47 \\
\hline PCRa (mg/L) & $3,13(2,9-11,25)$ & \multirow{3}{*}{53} \\
\hline Albúmina $(\mathrm{g} / \mathrm{dL})$ & $3,68(0,48)$ & \\
\hline Proteínas Totales (g/dL) & $6,78(0,68)$ & \\
\hline Colesterol Total (mg/dL) & $166,68(49,95)$ & 51 \\
\hline Triglicéridos (mg/dL) & $121,98(67,44)$ & 53 \\
\hline Colesterol H.D.L. (mg/dL) & $57,64(26,79)$ & 53 \\
\hline Colesterol L.D.L. (mg/dL) & $85,41(33,53)$ & 46 \\
\hline
\end{tabular}

Tabla 2: Características de la muestra al inicio del estudio 


\begin{tabular}{|c|c|c|c|c|c|}
\hline & & & Inicio & Año & $p^{*}$ \\
\hline OBESIDAD & \multicolumn{2}{|c|}{ I.M.C. > $30 \mathrm{Kg} / \mathrm{m}^{2}$} & $24,5 \%(13)$ & $24,5 \%(13)$ & 1 \\
\hline \multirow[b]{2}{*}{ DISLIPEMIA } & \multicolumn{2}{|c|}{ Triglicéridos $\geq 150 \mathrm{mg} / \mathrm{dL}$} & $22,6 \%(12)$ & $32,1 \%(17)$ & 0,332 \\
\hline & $\begin{array}{l}\text { Colesterol } \\
\text { H.D.L. }\end{array}$ & $\begin{array}{l}<40 \mathrm{mg} / \mathrm{dL} \text { en hombres } \\
<50 \mathrm{mg} / \mathrm{dL} \text { en mujeres }\end{array}$ & $32,1 \%(17)$ & $30,02 \%(16)$ & 1 \\
\hline \multirow[t]{2}{*}{ HIPERTENSIÓN ARTERIAL } & \multirow{2}{*}{$\begin{array}{l}\text { Tensión } \\
\text { arterial }\end{array}$} & $\begin{array}{l}\geq 130 \mathrm{~mm} \text { de } \mathrm{Hg} \text { sistólica } \\
\geq 85 \mathrm{~mm} \text { de } \mathrm{Hg} \text { diastólica }\end{array}$ & \multirow[t]{2}{*}{$73,6 \%(39)$} & \multirow[t]{2}{*}{$66 \%(35)$} & \multirow[t]{2}{*}{0,454} \\
\hline & & Hipertenso en tratamiento & & & \\
\hline GLUCEMIA & $\begin{array}{l}\text { Glucemia en } \\
\text { ayunas }\end{array}$ & $\begin{array}{l}\geq 100 \mathrm{mg} / \mathrm{dL} 0 \\
\text { Diabético en tratamiento }\end{array}$ & $28,3 \%(15)$ & $45,3 \%(24)$ & 0,012 \\
\hline \multicolumn{3}{|l|}{ SÍNDROME METABÓLICO } & $22,6 \%(12)$ & $32,1 \%(17)$ & 0,18 \\
\hline
\end{tabular}

Tabla 3: Características del Síndrome Metabólico en el primer año en Diálisis Peritoneal

nefropatía diabética en el 53,3\% ( $n=9)$ de los casos. Al año, el porcentaje de pacientes con una glucemia superior a $100 \mathrm{mg} / \mathrm{dL}$ aumentó de forma significativa al $32,1 \%(n=17),(p=0,012)$.

Treinta y tres pacientes $(62,3 \%)$ presentaron un IMC superior a $25 \mathrm{Kg} / \mathrm{m}^{2}$ (13 con obesidad franca y 20 con sobrepeso). No se encontró ningún paciente malnutrido (IMC $<20 \mathrm{Kg} / \mathrm{m}^{2}$ ). Al año, no se encontraron diferencias significativas en el IMC $(p=1)$. Sin embargo, sí hallamos significación cuando analizamos el peso al inicio $(71,13 \pm 13,49)$ y al año en DP $(74,07+14,41)$ con $p<0,001$. El aumento de peso medio de cada paciente fue de 2,94 $\pm 3,71 \mathrm{Kg}$.

El $68,5 \%(n=37)$ de los pacientes presento hipercolesterolemia (valores de colesterol $>200 \mathrm{~mL} / \mathrm{mg} 0$ en tratamiento con estatinas), 22,6\% ( $n=12)$ hipertrigliceridemia e hiperlipemia combinada el $25 \%$. El $32,1 \%(n=17)$ presentó bajos niveles de colesterol HDL. De ellos 64,2\% ( $n=34)$ estaban en tratamiento con estatinas y ninguno tomaba omega 3 . El resto es- taba en tratamiento dietético. Cuando analizamos los factores que intervienen en el diagnóstico del SM, la hipertrigliceridemia aumentó al año al 32,1\% ( $n=17)$, mientras que disminuyó al 30,02\% ( $n=16)$ los pacientes que presentaron niveles bajos de colesterol HDL. Ninguno de los factores tuvo significación.

Sobre la hipertensión arterial, al inicio del programa la presentaron 39 pacientes $(73,6 \%$ ) y al año 35 pacientes $(66 \%)$ aunque no hubo significación estadística $(p=0,454)$.

El número de factores de riesgo que presentaban los pacientes se muestra en la tabla 4, y aunque no hemos encontrado significación ( $p=0,213)$, con el tiempo aumentan el número de factores que presentan los pacientes.

La supervivencia de los pacientes con SM fue de 45,2 meses (IC (95\%) 42,09-52,67) frente a 47,38 meses $(36,56-53,85)$ en aquellos que no lo padecían. No hubo diferencia significativa $(p=0,990)$ como se muestra en la figura 5.

\begin{tabular}{|l|c|c|c|}
\hline & & Inicio & Año \\
\hline & 0 & $11,3 \%(6)$ & $11,3 \%(6)$ \\
\hline \multirow{2}{*}{ FACTORES DE RIESG0 } & 1 & $32,1 \%(17)$ & $32,1 \%(17)$ \\
& 2 & $34 \%(18)$ & $24,5 \%(13)$ \\
\hline & 3 & $11,3 \%(6)$ & $15,1(8)$ \\
\hline
\end{tabular}

Tabla 4: Número de factores de riesgo que presentaron los pacientes 


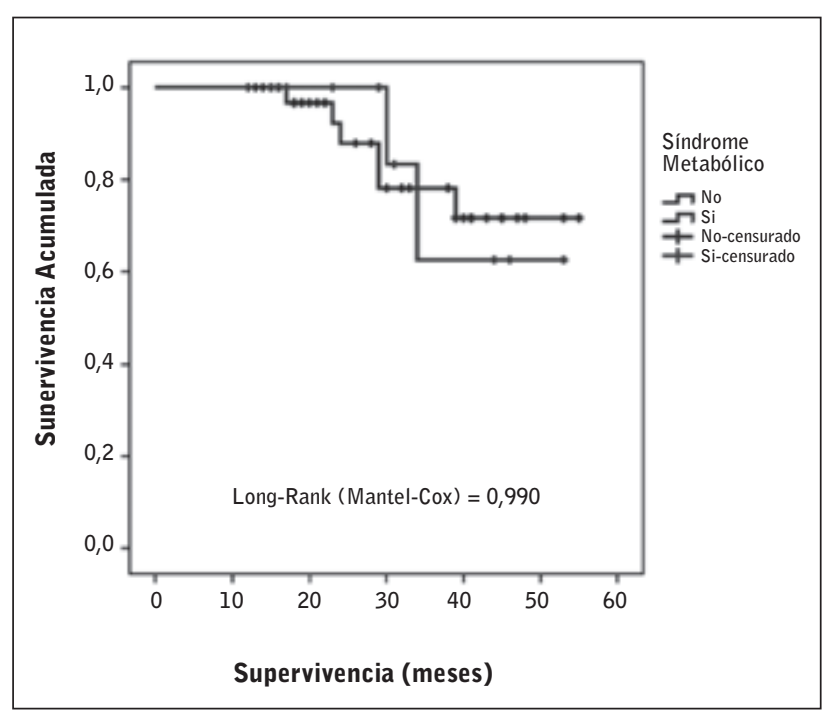

Figura 5. Síndrome Metabólico y Supervivencia de los pacientes en Diálisis Peritoneal

\section{Discusión}

En la población general la prevalencia del SM se estima por encima del $20 \%$ y en España oscila entre el $19,3 \%$ (criterios $0 \mathrm{MS})^{10}$ y el $15,5 \%$ (criterios EGIR) ${ }^{11}$. En nuestra muestra estos porcentajes están en el 22,6\% al inicio del programa de DP y aumentan al año de estancia, aunque no alcanzan el $53,3 \%$ de los pacientes que se han descrito en otros estudios ${ }^{1}$.

Esto puede deberse a que la DP, a pesar de ser una alternativa eficaz en el tratamiento de la ERC, puede provocar una sobrecarga de glucosa, lo que conlleva una serie de efectos metabólicos (diabetes, hiperlipemia, etc.) que implican ganancia de peso y tendencia a la obesidad ${ }^{12}$.

Por otro lado, la absorción de glucosa contribuye también a que los pacientes en DP presenten concentraciones de triglicéridos elevados ${ }^{13}$, datos que se corresponden con los obtenidos en nuestra población donde un 22,6\% presenta este factor de riesgo cardiovascular aumentando al $32,1 \%$ al año.

Durante el estudio, comprobamos como aumentó de forma significativa el porcentaje de pacientes cuyos niveles de glucemia eran superiores a $100 \mathrm{mg} / \mathrm{dL}$. Además, Szeto et $\mathrm{al}^{14}$, publicaron recientemente que la aparición en el primer mes de hiperglucemia en los pacientes que inician DP y no eran diabéticos previamente, tenían menor supervivencia de la técnica.
Dentro de los criterios utilizados para diagnosticar el SM, el perímetro abdominal se ha mostrado muy eficaz para predecir los eventos cardiovasculares. Sin embargo, en el caso de los pacientes en DP este no es valorable debido a la distención que pueden producir los líquidos de diálisis. Se ha propuesto el IMC para su valoración, ya que aunque menos específico, se correlaciona bien con el riesgo cardiovascular ${ }^{1}$. En nuestra muestra el $24,5 \%$ de los pacientes era obeso, y alcanzamos $63,5 \%$ si consideramos también a los que tienen sobrepeso. Ambos porcentajes son muy elevados si tenemos en cuenta que la obesidad también contribuye al deterioro de la función renal ${ }^{15}$.

A pesar de que en la población general los parámetros antropométricos son los que tienen mayor impacto en el diagnóstico del $\mathrm{SM}^{16}$, en nuestra muestra la hipertensión arterial fue el más prevalente (73,6\% de los pacientes).

Estudios epidemiológicos han demostrado que el SM incrementa el riesgo de complicaciones crónicas de la DM y se asocia a una mayor prevalencia de enfermedad cardiovascular, incrementando la mortalidad ${ }^{16}$. Sin embargo, estos estudios adquieren significación a largo plazo aumentando el riesgo cardiovascular tras una media de 9 años ${ }^{2}$, por lo que no es de extrañar que en este estudio de sólo un año de evolución no hayamos encontrado significación.

Una vez analizada la situación de nuestros pacientes así como la bibliografía existente sobre el SM, podemos afirmar que el papel de Enfermería en su prevención y tratamiento es fundamental ${ }^{18}$, ya que podemos actuar sobre todos los factores que definen este síndrome.

Sobre la obesidad y la dislipemia, podemos actuar a través de diagnósticos Enfermeros como "Desequilibrio nutricional por exceso", utilizando las encuestas dietéticas como una herramienta eficaz para obtener información sobre hábitos alimentarios no recomendables por favorecer el aumento de los lípidos y la glucemia.

Para corregir la hiperglucemia y la obesidad, debemos adaptar la actividad física a las características de nuestros pacientes. Problemas relacionados con la ERC como el dolor óseo, anemia, astenia, etc. hacen con frecuencia, que los pacientes lleven una vida sedentaria, por lo debemos idear pequeñas ac- 
tividades diarias, consensuadas con las familia, que favorezcan el ejercicio físico y se conviertan en rutinas habituales.

El "Déficit de Conocimientos" debe ser evaluado de forma sistemática, quizás con mayor frecuencia, ya que los resultados obtenidos indican que debemos reforzar las intervenciones sobre los conocimientos dietéticos y los hábitos cardiosaludables. De igual forma, las actividades relacionadas con el cumplimiento del tratamiento: manejo de hipotensores, medidas higiénicas, etc. para el control de la tensión arterial, deben ser una actividad constante en nuestra consulta de DP.

En definitiva, hemos comprobado la importancia que tiene establecer diagnósticos de Enfermería adecuados a los problemas de salud que presentan nuestros pacientes, así como reevaluar de forma frecuente y exhaustiva las intervenciones que diseñamos, con el fin de obtener los resultados esperados. En nuestra población, no hemos conseguido mejorar las características que definen el SM, e incluso en algunos casos, estas han empeorado al año, lo que nos ha obligado a plantearnos actuaciones más efectivas, para mejorar los resultados obtenidos.

Como conclusión, podemos decir que en nuestra unidad tenemos un elevado porcentaje de pacientes diagnosticados o con riesgo de padecer SM y esta situación parece aumentar al año de DP.

Los pacientes con SM al comienzo del estudio tuvieron una supervivencia menor que el resto de la población estudiada, aunque sin llegar a ser significativa. Pensamos que puede deberse al tamaño muestral y a que los FRCV aumentan la mortalidad a medio-largo plazo y no en el primer año de tratamiento.

Debemos evaluar y corregir los diagnósticos de enfermería definidos así como las intervenciones que proponemos en la consulta, ya que creemos posible disminuir prevalencia del SM en nuestra población.

Recibido: Septiembre 2011

Revisado: Octubre 2011

Modificado: Octubre 2011

Aceptado: Octubre 2011

\section{Bibliografía}

1. Philip Kam-Tao Li, Bonnie Ching-Ha Kwan, Cheuk Chun Szeto et al. Metabolic syndrome in peritoneal dialysis patients. NDT Plus (2008) 4: 206-214.

2. Martínez A. Síndrome Metabólico y nefropatía. Nefrología.2008; Supl. 3:33-37.

3. Hernández D, Álvarez A, Armas AM et al. Síndrome metabólico y donación renal de vivo: ¿este síndrome contraindica la donación? Nefrología 2009; 29(1):20-29.

4. Del Álamo A. Síndrome Metabólico. Guías clínicas 2005; 5(2). http://www.fisterra.com/guias2/Smetabolico.asp\#que.

5. Gami AS, Witt BJ, Howard DE, et al. Metabolic syndrome and risk of incident cardiovascular events and death: a systematic review and meta-analysis of longitudinal studies. J Am Coll Cardiol 2007; 49:403-14.

6. Reaven GM. Role of insulin resistance in human disease. Diabetes. 1988; 37:1595-1607.

7. Executive summary of the third report of the National Cholesterol Education program (NCEP). Expert Panel on detection, evaluation and treatment of high blood cholesterol in adults (Adult treatment panel III). JAMA 2001; 285:2486-97.

8. Sociedad Española para el estudio de la Obesidad (SEED0). Consenso SEEDO 2000 para la evaluación del sobrepeso y la obesidad y el establecimiento de criterios de intervención terapéutica. Med Clin (Barc) 2000; 115:587-97.

9. Códigos EDTA 1994-1995 y su agrupación, extraídos del Informe 2006. Módulo Básico. Subsistema de Insuficiencia Renal Crónica. Servicio Andaluz de Salud. Junta de Andalucía.

10. Serrano M, Ascaso JF, Blázquez E et al. Resistencia a la insulina y su implicación en múltiples factores de riesgo asociados a diabetes tipo 2. Med Clin (Barc) 2002; 119:458-63.

11. The european Group for the study of insulin resistance (EGIR). Frecuency of the Who metabolic syn- 
drome in European cohorts, and an alternative definition of an insulin resistance syndrome. Diabetes Metab 2002; 28:364-76.

12. Diez $\mathrm{J}$ et al. Diabetes, hiperlipemia y obesidad en una unidad de diálisis peritoneal: estudio descriptivo transversal.Nefrología.1995; XV (2):156-162.

13. Feldman HA, Singer I. Endocrinology and metabolism in uremia and dialysis: a clinical review. Medicine (Baltimore). 1974; 54:345-380.

14. Szeto CC, Chow KM, Kwan BC, et al. New-onset hyperglycemia in nondiabetic Chinese patients started on peritoneal dialysis. Am J Kidney Dis. 2007; 49:524-532.
15. Tozawa $M$ et al. Influence of smoking and obesity on the development of proteinuria. Kidney Int 2002; 62:956-962.

16. Ascaso JF et al. Diagnóstico del síndrome metabólico. Adecuación de los criterios diagnósticos en nuestro medio. Recomendaciones del foro HDL. Resumen ejecutivo. Rev Clin Esp 2006; 11:576-582.

17. Ford ES. Risk for all-cause mortality, cardiovascular disease, and diabetes associated with metabolic síndrome: a summary of the evidence. Diabetes Care. 2005; 28:1769-78.

18. Iborra et al. Síndrome metabólico en hemodiálisis. Actuación Enfermera. Rev. Soc. Esp. Enferm Nefrol. 2008; 11 (2): 89-94. 